\title{
Perfil del patrón de sueño en deportistas universitarios*
}

\author{
Pedro Carazo Vargas ${ }^{* *}$ \\ José Moncada Jiménez ${ }^{* * *}$
}

\author{
Recibido: enero 20 del 2021
}

Aceptado: febrero og del 2021

Citar como:

Carazo Vargas, P. y Moncada Jiménez, J. (2021). Perfil del patrón de sueño en deportistas universitarios. Revista de Investigación Cuerpo, Cultura y Movimiento, 11(1). https://doi.org/10.15332/2422474X.6469

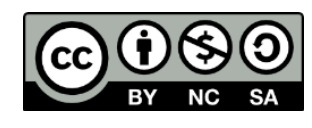

\section{Resumen}

El objetivo de la presente investigación es describir y analizar los patrones de sueño de hombres y mujeres deportistas universitarios practicantes de fútbol, baloncesto, voleibol, atletismo, natación y taekwondo. Participaron 114 deportistas ( 56 hombres, 58 mujeres), quienes durante 7 días utilizaron un acelerómetro modelo wGT3X-BT al momento de dormir. Todas las mediciones fueron realizadas al inicio del periodo de preparación de los deportistas. Se presentaron interacciones entre el sexo y el deporte para la eficiencia de sueño $(p=0.006)$, el tiempo en despertar $(p=0.016)$, el tiempo en cama $(p=0.048)$ y el

\footnotetext{
* Artículo resultado de investigación, financiamiento propio. La recolección de datos para la investigación se efectuó entre enero y febrero del 2020.

** Doctor en Ciencias del Movimiento Humano; investigador del Centro de Investigación en Ciencias del Movimiento Humano, Universidad de Costa Rica. Docente de la Escuela de Educación Física y Deportes de la Universidad de Costa Rica. Correo electrónico: pedro.carazo@ucr.ac.cr; ORCID: https://orcid.org/0000-0002-5374-7031 *** Doctor en Ciencias Biomédicas; investigador del Centro de Investigación en Ciencias del Movimiento Humano, Universidad de Costa Rica. Docente de la Escuela de Educación Física y Deportes de la Universidad de Costa Rica. Correo electrónico: jose.moncada@ucr.ac.cr; ORCID; https://orcid.org/0000-0001-9807-5163
} 
tiempo de sueño ( $\mathrm{p}=$ o.006). La latencia, así como la cantidad y la duración de los despertares, no difieren según el sexo ni el deporte practicado. Independientemente del deporte, los hombres presentan una mayor cantidad $(\mathrm{p}=0.046)$ y duración $(\mathrm{p}=0.002)$ de despertares que las mujeres. Se observaron valores de eficiencia y duración de sueño inferiores a los deseados. Se recomienda vigilar los patrones de sueño de los deportistas universitarios, ya que las obligaciones académicas podrían afectar sus hábitos de sueño.

Palabras clave: deporte, estudiantes, rendimiento.

\section{Sleep pattern profile in university athletes}

\section{Abstract}

The purpose of this research is to describe and analyze the sleep patterns of male and female university athletes practicing soccer, basketball, volleyball, athletics, swimming, and taekwondo. A total of 114 athletes ( 56 men, 58 women) participated, who used a wGT3X-BT accelerometer during 7 days while sleeping. All measurements were taken at the beginning of the athletes' preparation period. Interactions between gender and sport were found for sleep efficiency $(\mathrm{p}=0.006)$, time in awakening $(\mathrm{p}=0.016)$, time in bed $(\mathrm{p}=0.048)$ and sleep time $(\mathrm{p}=$ o.006). The latency, as well as the number and duration of awakenings, did not differ between gender and sport. Regardless of the sport, men showed a greater number $(\mathrm{p}=0.046)$ and duration $(\mathrm{p}=0.002)$ of awakenings than women. Sleep efficiency and sleep duration values were lower than desired. It is recommended to monitor the sleep patterns of university athletes, since academic obligations could affect their sleep habits.

Keywords: sport, students, performance. 


\section{Introducción}

Uno de los principales objetivos de los deportistas es el de maximizar su rendimiento en las competiciones. Para esto, se someten a un proceso de preparación que requiere dedicación y esfuerzo. Con el fin de lograr esta meta, la población deportista universitaria debe organizar su tiempo para cumplir paralelamente con las exigencias de su disciplina deportiva y sus deberes académicos. La cantidad de obligaciones que debe atender esta población puede conducirla a alterar negativamente sus patrones de sueño, elemento que debe llamar a la reflexión para no afectar la salud, el desempeño deportivo ni el rendimiento académico de estos deportistas.

Es relevante señalar que el sueño es una necesidad biológica imprescindible para el adecuado funcionamiento del organismo de las personas (Watson et ál., 2015). Las variables que permiten conocer la estructura del sueño de las personas incluyen la latencia, definida como el tiempo aproximado que necesita una persona para dormirse; la eficiencia de sueño, usualmente reportada como un porcentaje, que es la cantidad de minutos que se pasa dormido dividido entre la cantidad total de minutos durante los que la persona estuvo en la cama. Otras variables incluyen el tiempo total en cama y dormido, así como la cantidad y la duración promedio de los despertares (ActiGraph, 2012).

El sueño ininterrumpido, con suficiente cantidad y calidad de horas, está asociado a múltiples beneficios, como, por ejemplo, el fortalecimiento de los procesos de memoria y aprendizaje (Van Der Werf et ál., 2009; Vyazovskiy, 2015). Las alteraciones en los patrones de sueño podrían afectar negativamente el funcionamiento del sistema nervioso y el sistema inmune (Fullagar et ál., 2015; Gómez-González et ál., 2012; Peake et ál., 2016). Existen datos que indican la prevalencia de insomnio (38\%), molestias por insomnio (36\%), dificultad para dormirse (18\%), dificultad 
para mantenerse dormido (42 \%) y despertarse súbitamente (21 \%) en países específicos como Estados Unidos, Australia, Alemania, Brasil, Taiwán, Islandia, y en regiones de la Unión Europea, en la Región Pacífico Occidental, la Región del Sureste de Asia y en América (Zhang et ál., 2019). Por lo tanto, los problemas asociados al sueño se convierten en un tema de salud pública global.

En conjunto, los problemas del sueño pueden impactar negativamente la calidad de vida de las personas, asociándoseles, por ejemplo, a un deterioro cognitivo o demencia, experiencias psicóticas, depresión, ansiedad y fatiga, entre otros (Reeve et ál., 2018; Xu et ál., 2020). Las personas deportistas no están exentas de presentar problemas de sueño y se ha descrito que la restricción del sueño perjudica la síntesis de proteínas musculares, la fuerza máxima isométrica, el rendimiento aeróbico y el rendimiento cognitivo (Ben Cheikh et ál., 2016; Keramidas et ál., 2018; Saner et ál., 2020; Skein et ál., 2013). Por el contrario, la extensión en la cantidad de horas que duermen los deportistas podría generarles beneficios en su desempeño deportivo (Mah et ál., 2011; Schwartz y Simon, 2015). Sin embargo, también se reconoce que existe una respuesta individual muy variable entre los atletas (Carazo-Vargas y MoncadaJiménez, 2020).

Se puede argumentar que el sueño representa un elemento de gran importancia para los deportistas, ya que puede disminuir el riesgo de aparición de enfermedades agudas y crónicas, así como evitar lesiones. Debido a la asociación entre el sueño y el desempeño cognitivo, la salud física y mental, los patrones positivos de sueño pueden tener un impacto efectivo en el rendimiento deportivo (Copenhaver y Diamond, 2017; Halson, 2014b). De esta manera, han sido numerosas las áreas en las que se ha estudiado la influencia del sueño en el deporte; algunas de ellas son, el efecto agudo de la pérdida de sueño, el impacto de restringir el sueño de 
manera prolongada, la relación con la aparición de lesiones, la extensión del sueño, la recuperación y las alteraciones en el estado de ánimo (Chennaoui et ál., 2015; Fullagar et ál., 2015; Fullagar et ál., 2015; Simpson et ál., 2017).

En consecuencia, para la búsqueda del rendimiento deportivo óptimo, se hace imprescindible conocer los patrones de sueño de los deportistas. Esto representa un factor adicional para considerar durante el monitoreo de la carga interna de los deportistas durante el proceso de entrenamiento (Halson, 2014a). No obstante, el interés por estudiar la cantidad y calidad del sueño en la población deportista es un tema reciente del que aún no se tiene suficiente evidencia (Fullagar et ál., 2015; Halson, 2014b). En este contexto, el objetivo de la presente investigación fue describir y analizar los patrones de sueño de hombres y mujeres deportistas universitarios practicantes de fútbol, baloncesto, voleibol, atletismo, natación y taekwondo.

\section{Metodología}

\section{Participantes}

Participaron voluntariamente 114 atletas (edad $=21.46 \pm 2.07$ años) provenientes de los equipos de representación de la Universidad de Costa Rica (UCR) de las disciplinas deportivas de atletismo $(\mathrm{n}=11)$, natación $(n=15)$, taekwondo $(n=13)$, baloncesto $(n=25)$, fútbol $(n=33)$ y voleibol $(\mathrm{n}=17)$. De las personas participantes, 56 eran hombres y 58 mujeres.

Los participantes se incluyeron en el estudio si pertenecían a uno de los equipos de representación de la UCR, se encontraban entrenando de manera regular y no consumían ningún tipo de medicamento para regular algún tipo de problema de sueño. El protocolo fue aprobado por el Comité ético científico de la Universidad de Costa Rica, código 838-B8-355; cada 
participante leyó y firmó el respectivo consentimiento informado del estudio.

\section{Procedimientos}

Las medidas antropométricas de talla $(\mathrm{cm})$ y peso corporal $(\mathrm{kg})$ fueron obtenidas con un estadiómetro y una báscula electrónica SECA modelo 256 dp (Chino, CA). Mediante un acelerómetro modelo wGT3X-BT y el software ActiLife 6 (ActiGraph ${ }^{\mathrm{TM}}$, Pensacola, FL), se determinó la latencia y la eficiencia del sueño, el tiempo total en cama y en sueño, así como la cantidad y la duración promedio de los despertares.

Todos los atletas que participaron en el estudio se encontraban entrenando al inicio de su periodo preparatorio de competición. A cada uno de ellos se le proporcionó un acelerómetro que se llevaron a sus casas, el cual tenían que utilizar para dormir durante 7 días. A los participantes se les solicitó no modificar su patrón habitual de sueño por la participación en el estudio, así como llevar una bitácora en la que debían anotar la hora en la que se acostaban a dormir y la hora en que despertaban. El acelerómetro se puso en la muñeca de la mano no dominante de los participantes, siguiendo el mismo protocolo establecido en otro estudio (Carazo-Vargas y Moncada-Jiménez, 2020).

\section{Análisis estadísticos}

La estadística descriptiva se presenta mediante promedios y desviaciones estándar $(\mathrm{M} \pm \mathrm{DE})$. Para evaluar la presencia de diferencias estadísticas entre los distintos promedios de las variables de estudio según el deporte practicado y el sexo se realizaron análisis de varianza Anova de dos vías para grupos independientes. A las interacciones significativas se les dio seguimiento mediante análisis post hoc de Bonferroni. Se empleó el 
paquete estadístico IBM-SPSS, versión 25 (IBM Corporation, Armonk, New York). La significancia estadística se estableció a priori en $\mathrm{p} \leq \mathrm{0.05}$.

\section{Resultados}

En la tabla 1 se presenta la estadística descriptiva de la edad, peso y estatura de las personas participantes en el estudio según el sexo y deporte practicado. Por su parte, en la tabla 2 se muestra la estadística descriptiva para cada una de las variables de sueño, en los seis deportes analizados, tanto para hombres como para mujeres. Solamente se presentó la exclusión de un participante, dado que durante la semana de mediciones sufrió una fractura en un brazo que le impidió continuar entrenando, por lo tanto, sus datos no se incluyeron en el análisis.

Tabla 1. Estadística descriptiva $(M \pm D E)$ de atletas universitarios por sexo y deporte

\begin{tabular}{|l|l|l|l|l|}
\hline \multicolumn{1}{|c|}{ Sexo } & \multicolumn{1}{|c|}{ Deporte } & Edad (años) & \multicolumn{1}{|c|}{ Peso (kg) } & $\begin{array}{c}\text { Estatura } \\
\text { (cm) }\end{array}$ \\
\hline $\begin{array}{l}\text { Hombres } \\
(\mathbf{n = 5 6 )}\end{array}$ & Atletismo $(\mathrm{n}=4)$ & $22.14 \pm 1.77$ & $67.74 \pm 8.12$ & $174.2 \pm 5.47$ \\
\hline & Natación $(\mathrm{n}=8)$ & $22.13 \pm 2.95$ & $82.55 \pm 19.70$ & $174.9 \pm 5.69$ \\
\hline & Taekwondo $(\mathrm{n}=7)$ & $21.43 \pm 1.90$ & $71.17 \pm 17.00$ & $175.6 \pm 8.27$ \\
\hline & Baloncesto $(\mathrm{n}=13)$ & $20.85 \pm 1.77$ & $82.69 \pm 15.30$ & $184.5 \pm 6.20$ \\
\hline & Fútbol (n=15) & $21.73 \pm 2.25$ & $70.90 \pm 4.95$ & $173.3 \pm 5.82$ \\
\hline & Voleibol (n=11) & $21.18 \pm 1.66$ & $77.55 \pm 9.15$ & $181.1 \pm 6.20$ \\
\hline & $\begin{array}{l}\text { Total hombres } \\
\text { (n=58) }\end{array}$ & $21.58 \pm 2.05$ & $75.43 \pm 12.37$ & $177.27 \pm 6.28$ \\
\hline
\end{tabular}

\begin{tabular}{|c|c|c|c|c|}
\hline \multirow[t]{5}{*}{$\begin{array}{l}\text { Mujeres }(n= \\
58)\end{array}$} & Atletismo $(\mathrm{n}=7)$ & $20.25 \pm 0.96$ & $67.60 \pm 12.8$ & $168.0 \pm 3.38$ \\
\hline & Natación $(\mathrm{n}=7)$ & $21.43 \pm 1.38$ & $61.98 \pm 7.76$ & $164.1 \pm 6.87$ \\
\hline & Taekwondo $(n=6)$ & $22.16 \pm 1.60$ & $62.55 \pm 16.20$ & $160.8 \pm 4.96$ \\
\hline & Baloncesto $(n=12)$ & $21.33 \pm 2.59$ & $64.93 \pm 11.10$ & $166.9 \pm 9.06$ \\
\hline & Fútbol $(\mathrm{n}=18)$ & $21.33 \pm 2.59$ & $58.59 \pm 9.42$ & $159.7 \pm 4.99$ \\
\hline & Voleibol $(n=6)$ & $21.67 \pm 1.21$ & $63.33 \pm 6.12$ & $166.7 \pm 6.62$ \\
\hline
\end{tabular}




\begin{tabular}{|l|l|l|l|l|}
\hline Sexo & \multicolumn{1}{|c|}{ Deporte } & Edad (años) & Peso (kg) & $\begin{array}{c}\text { Estatura } \\
\text { (cm) }\end{array}$ \\
\hline & Total mujeres $(\mathrm{n}=56)$ & $21.36 \pm 1.72$ & $63.16 \pm 10.57$ & $164.37 \pm 5.98$ \\
\hline & Total general $(\mathrm{n}=114)$ & $21.47 \pm 1.88$ & $69.30 \pm 11.47$ & $170.82 \pm 6.13$ \\
\hline
\end{tabular}

Fuente: elaboración propia.

Revista de Investigación Cuerpo, Cultura y Movimiento

ISSN: 2248-4418 | e-ISSN: 2422-474X | DOI: https://doi.org/10.15332/2422474X

Vol. 11 N. ${ }^{\circ} 1$ | enero-junio de 2021 
Tabla 2. Estadística descriptiva $(M \pm D E)$ de las variables de sueño en atletas universitarios $(n=114)$

\begin{tabular}{|c|c|c|c|c|c|c|c|c|}
\hline Deporte & Sexo & $\begin{array}{l}\text { Latencia } \\
\text { (min) }\end{array}$ & $\begin{array}{c}\text { Eficiencia } \\
\text { (\%) }\end{array}$ & $\begin{array}{l}\text { Tiempo en } \\
\text { cama (min) }\end{array}$ & $\begin{array}{c}\text { Tiempo } \\
\text { sueño (min) }\end{array}$ & $\begin{array}{l}\text { Tiempo } \\
\text { despertar } \\
\text { (min) }\end{array}$ & $\begin{array}{l}\text { Despertares } \\
\text { (unidades) }\end{array}$ & $\begin{array}{l}\text { Duración } \\
\text { Despertares } \\
\text { (min) }\end{array}$ \\
\hline \multirow[t]{2}{*}{ Atletismo } & Hombres & $3.0 \pm 3.2$ & $84.6 \pm 3.5$ & $466.2 \pm 26.4$ & $355 \cdot 3 \pm 47 \cdot 4$ & $71.3 \pm 34.1$ & $22.5 \pm 6.7$ & $3.1 \pm 1.2$ \\
\hline & Mujeres & $3 \cdot 3 \pm 1.8$ & $82.6 \pm 9.0$ & $429.6 \pm 32.6$ & $392.6 \pm 29.8$ & $70.4 \pm 17.0$ & $23.8 \pm 3.6$ & $2.9 \pm 0.4$ \\
\hline \multirow[t]{2}{*}{ Natación } & Hombres & $4.2 \pm 4.5$ & $87.6 \pm 4.3$ & $448.6 \pm 36.7$ & $338.6 \pm 37.4$ & $105.7 \pm 37.2$ & $28.7 \pm 8.2$ & $3.7 \pm 0.8$ \\
\hline & Mujeres & $4.1 \pm 2.1$ & $75.6 \pm 7.8$ & $482.4 \pm 37.3$ & $419.1 \pm 29.9$ & $59.2 \pm 24.1$ & $21.3 \pm 9.0$ & $2.8 \pm 0.6$ \\
\hline \multirow[t]{2}{*}{ Taekwondo } & Hombres & $5.0 \pm 5.2$ & $82.9 \pm 6.8$ & $450.5 \pm 62.2$ & $381.9 \pm 51.4$ & $63.8 \pm 19.3$ & $22.0 \pm 3.6$ & $2.8 \pm 0.6$ \\
\hline & Mujeres & $4.6 \pm 3.1$ & $84.9 \pm 4.6$ & $467.8 \pm 64.2$ & $386.8 \pm 51.9$ & $76.3 \pm 32.3$ & $26.0 \pm 8.5$ & $3.0 \pm 0.7$ \\
\hline \multirow[t]{2}{*}{ Baloncesto } & Hombres & $4.4 \pm 3.2$ & $77.1 \pm 6.5$ & $442.8 \pm 42.6$ & $338.7 \pm 36.4$ & $99.4 \pm 30.4$ & $25.7 \pm 8.1$ & $4.5 \pm 1.2$ \\
\hline & Mujeres & $3.7 \pm 2.5$ & $86.1 \pm 4.1$ & $490.6 \pm 62.5$ & $421.8 \pm 49.8$ & $65.7 \pm 26.5$ & $22.4 \pm 6.2$ & $3.0 \pm 0.7$ \\
\hline \multirow[t]{2}{*}{ Fútbol } & Hombres & $5.4 \pm 4.2$ & $76.1 \pm 6.9$ & $481.6 \pm 50.1$ & $371.3 \pm 36.5$ & $105.1 \pm 34.9$ & $29.1 \pm 7.2$ & $3.7 \pm 0.9$ \\
\hline & Mujeres & $3.8 \pm 3.1$ & $85.9 \pm 2.7$ & $456.8 \pm 60.8$ & $391.4 \pm 49.4$ & $61.1 \pm 15.9$ & $23.0 \pm 6.7$ & $2.8 \pm 0.4$ \\
\hline \multirow[t]{2}{*}{ Voleibol } & Hombres & $8.2 \pm 8.7$ & $82.1 \pm 5.0$ & $492.5 \pm 63.1$ & $405.6 \pm 61.6$ & $90.5 \pm 33.7$ & $26.2 \pm 6.2$ & $3.1 \pm 0.6$ \\
\hline & Mujeres & $4.4 \pm 3.1$ & $85.8 \pm 4.4$ & $455 \cdot 9 \pm 35 \cdot 9$ & $392.5 \pm 44.8$ & $64.1 \pm 21.9$ & $20.4 \pm 5.3$ & $2.9 \pm 0.6$ \\
\hline \multirow[t]{3}{*}{ General } & Hombres & $5.0 \pm 4.8$ & $81.7 \pm 5.5$ & $463.7 \pm 46.9$ & $365.2 \pm 45.1$ & $89.3 \pm 31.6$ & $25.7 \pm 6.7$ & $3.5 \pm 0.9$ \\
\hline & Mujeres & $4.0 \pm 2.6$ & $83.5 \pm 5.4$ & $463.8 \pm 48.9$ & $400.7 \pm 42.6$ & $66.1 \pm 23.0$ & $22.8 \pm 6.6$ & $2.9 \pm 0.6$ \\
\hline & Total & $4.5 \pm 3.7$ & $82.6 \pm 5.5$ & $463.8 \pm 47.9$ & $383.0 \pm 43.9$ & $77 \cdot 7 \pm 27 \cdot 3$ & $24.6 \pm 6.6$ & $3.2 \pm 0.7$ \\
\hline
\end{tabular}

Fuente: elaboración propia.

Revista de Investigación Cuerpo, Cultura y Movimiento

ISSN: 2248-4418 | e-ISSN: 2422-474X | DOI: https://doi.org/10.15332/2422474X

Vol. 11 N. ${ }^{\circ} 1$ | enero-junio de 2021 
El análisis estadístico inferencial determinó que la latencia de sueño no diferente según el sexo y el deporte practicado $(\mathrm{p}=0.82)$. Se presentó una interacción entre el sexo del participante y el deporte practicado para la eficiencia de sueño $(\mathrm{p}=$ o.006). El análisis de efectos simples mostró que existen diferencias en la eficiencia de sueño entre los hombres y las mujeres practicantes de natación, fútbol y baloncesto (tabla $2, \mathrm{p}<0.05$ ). El tiempo en despertar fue distinto según el sexo y el deporte practicado ( $p=0.016)$. Los hombres que practican natación, baloncesto y fútbol permanecen una mayor cantidad de minutos durmiendo antes de despertarse por primera vez respecto a las mujeres que practican estas disciplinas (tabla 2, $\mathrm{p}<0.05$ ). Asimismo, los hombres practicantes de taekwondo mostraron un menor tiempo en despertar que los futbolistas (tabla 2, p < 0.05).

Existen diferencias en el tiempo en cama entre los sexos según el deporte practicado $(\mathrm{p}=0.048)$. Las mujeres que practican baloncesto permanecen en cama una mayor cantidad de minutos que los hombres basquetbolistas (tabla 2, p < 0.05). El tiempo en que efectivamente permanecen en sueño también difiere según el sexo y el deporte practicado $(\mathrm{p}=0.006)$. Hay diferencias entre hombres y mujeres que practican natación y baloncesto; también entre los hombres que practican voleibol respecto de los que practican natación y baloncesto (tabla $2, \mathrm{p}<0.05$, figura 1 ). 
Figura 1. Interacciones estadísticas entre el sexo y el deporte en las variables relacionadas al sueño. a) Eficiencia, b) Tiempo en despertar, c) Tiempo en cama, d) Tiempo durmiendo.

a)
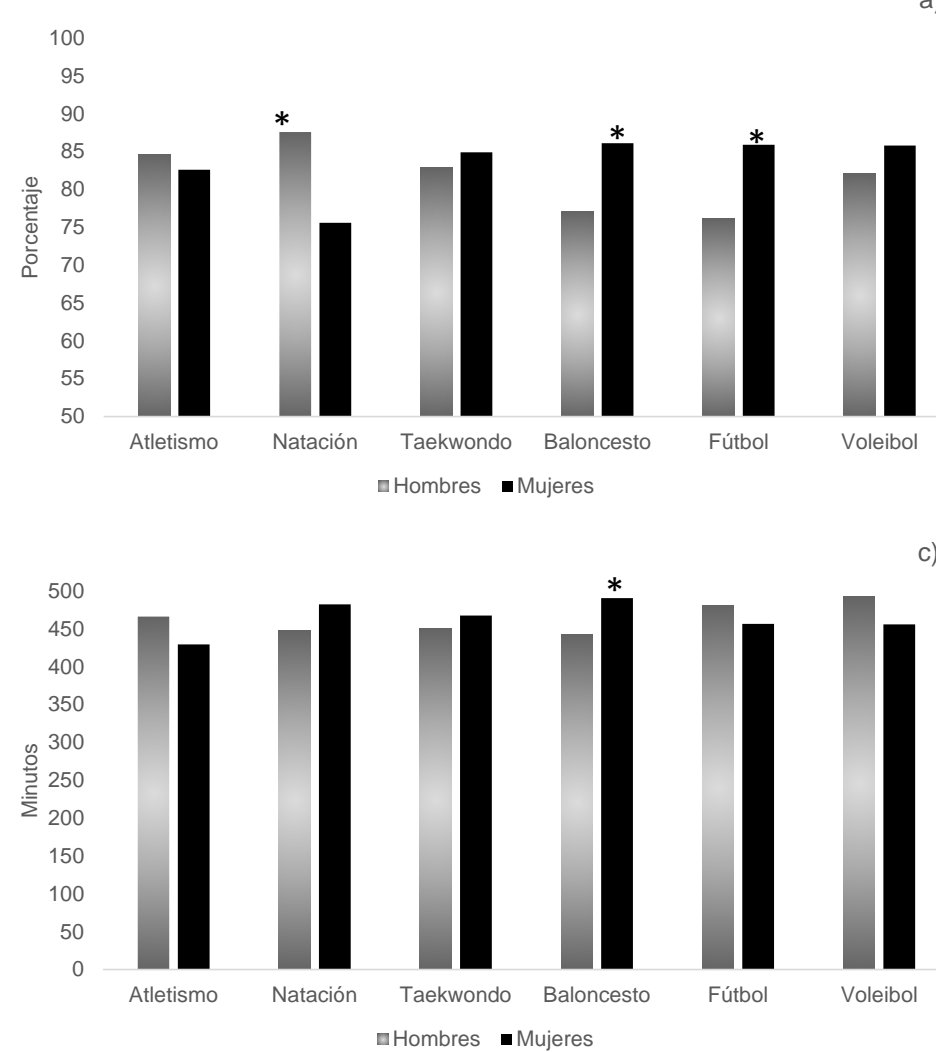

b)
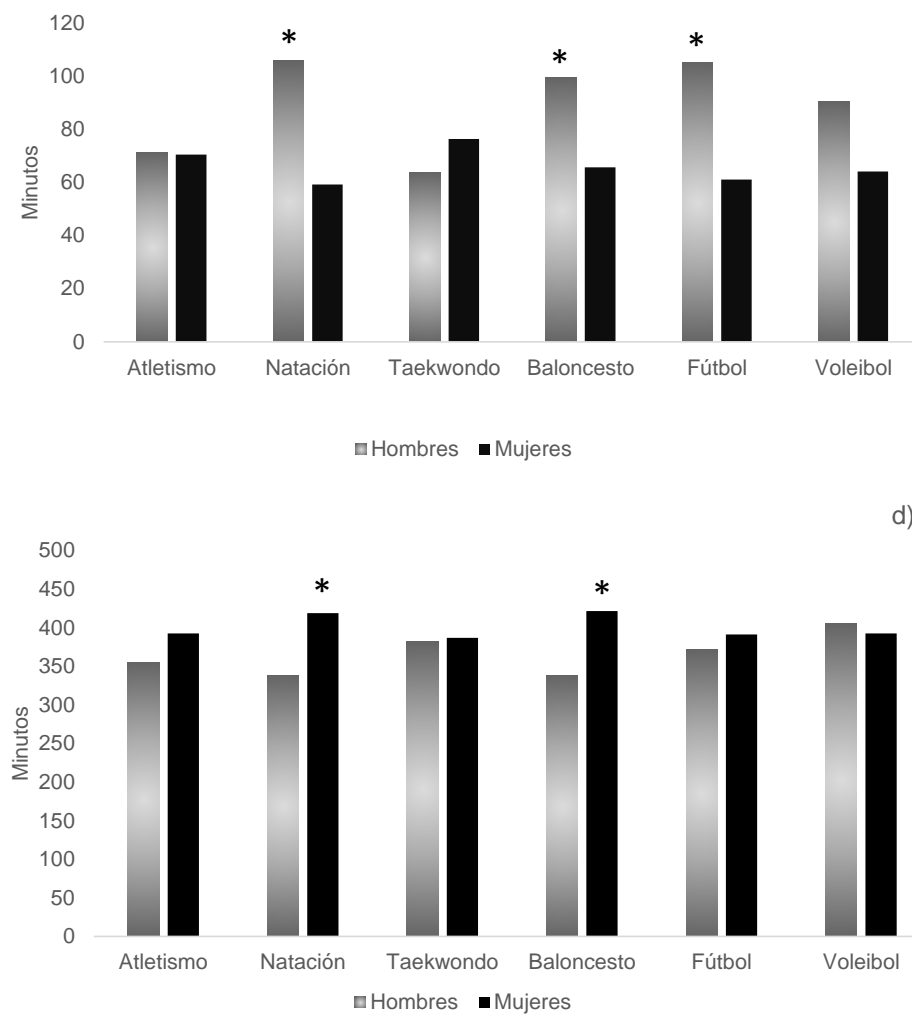

Nota: * refleja diferencias entre hombres y mujeres según el deporte practicado $(p<0.05)$.

Fuente: elaboración propia. 
$\mathrm{Al}$ analizar la cantidad y la duración de los despertares, se encontró que el deporte practicado no tiene influencia en estas variables. Únicamente se identificó la presencia de una mayor cantidad de despertares según el sexo, en donde, en promedio, los varones presentan una mayor cantidad de despertares que las mujeres ( $25.71 \pm 0.93$ vs. $22.82 \pm 1.08, p=0.046)$. De manera congruente, se observó una diferencia en la duración en los despertares según el sexo, siendo mayor la cantidad de minutos de estos despertares en los hombres respecto de las mujeres (3.41 $\pm 0.10 \mathrm{~min}$. vs. $2.92 \pm 0.12$ min., $\mathrm{p}=0.002$ ).

\section{Discusión}

El presente estudio permitió caracterizar los patrones de sueño de hombres y mujeres deportistas universitarios practicantes de fútbol, baloncesto, voleibol, atletismo, natación y taekwondo. Los valores reportados son útiles para valorar los rasgos de sueño de poblaciones similares, quienes además de prepararse para rendir deportivamente, deben atender importantes responsabilidades académicas.

Según los resultados obtenidos, no se identifica algún indicio que sugiera que los patrones de sueño se vean afectados de alguna manera por las características del entrenamiento que requieren los distintos deportes. De esta manera, las variaciones en los patrones de sueño en el transcurso de un periodo de preparación podrían deberse a las modificaciones tanto en el volumen como en la intensidad de los regímenes de preparación a los que se ven sometidos los deportistas (Taylor et ál., 1997; Walsh et ál., 2019), y no a la orientación de los objetivos de entrenamiento sobre los que se realizan las adaptaciones.

En la eficiencia del sueño, el tiempo en despertar por primera vez, el tiempo en cama y dormidos, se presentaron diferencias entre hombres y 
mujeres practicantes de natación, baloncesto y fútbol. Asimismo, en este estudio se observó, en general, un mejor perfil de sueño en las mujeres en comparación con el de los hombres. Este resultado coincide con los reportado por Leeder et ál. (2012), quienes concluyeron que las mujeres muestran una mejor eficiencia de sueño que los varones. Dichos resultados refuerzan la evidencia previa que sostiene la presencia de diferencias en los patrones de sueño entre hombres y mujeres, aunque las razones de estas discrepancias permanecen aún sin ser explicadas, y es probable que se deban a factores de índole social u hormonal (Carrier et ál., 2017; Ketema et ál., 2006; Mong y Cusmano, 2015).

La eficiencia de sueño registrada por los participantes, independientemente del deporte practicado, concuerda con los valores reportados en estudios anteriores, sugiriendo que los deportistas tienden a mostrar patrones de sueño menos eficientes que las personas que no practican deporte competitivo y que los atletas tienden a presentar problemas de sueño (Tuomilehto et ál., 2017; Leeder et ál., 2012; Sargent et ál., 2014; Walsh et ál., 2019).

Aunque debe considerarse que los monitores de actividad pueden subestimar la duración del sueño (Sargent et ál., 2016), llama la atención que el tiempo efectivo que los deportistas dormían independientemente del sexo y el deporte practicado (6.38 \pm 0.73 horas), se encontrara por debajo de la recomendación de horas de sueño para personas con edades entre los 18 y 25 años (Walsh et ál., 2020).

Es fundamental lograr que los deportistas universitarios logren estructurar sus horarios para cumplir sus obligaciones académicas y deportivas sin reducir la cantidad de horas que duermen, ya que dormir una menor cantidad de horas incrementa la posibilidad de que los deportistas jóvenes se lesionen (Milewski et ál., 2014). 
$\mathrm{Al}$ reconocer la importancia del sueño en el desempeño deportivo y la salud (Fullagar et ál., 2015; Halson, 2014b), la eficiencia y la cantidad de horas de sueño registradas deben llamar la atención del personal responsable del entrenamiento de los deportistas universitarios; esto con el objetivo de reforzar la comprensión de la importancia del sueño para el éxito deportivo y el bienestar de las personas, e incentivar hábitos saludables para mejorarlo.

Para futuras investigaciones se deberían describir y comparar los patrones de sueño durante diferentes etapas de la planificación del entrenamiento, con el propósito de determinar si las cargas de trabajo afectan positiva o negativamente los patrones de sueño de los deportistas.

\section{Referencias}

ActiGraph. (2012). ActiLife 6 User's Manual.

http://s3.amazonaws.com/actigraphcorp.com/wpcontent/uploads/2018/02/22094126/GT3X-wGT3X-Device-Manual-110315.pdf

Ben Cheikh, R., Latiri, I., Dogui, M. y Ben Saad, H. (2016). Effects of one night sleep deprivation on selective attention and isometric force in adolescent karate athletes. Journal of sports medicine and physical fitness, 57(6), 752-759. https://www.minervamedica.it/en/journals/sports-med-physicalfitness/article.php?cod=R40Y2017No6A0752

Carazo-Vargas, P. y Moncada-Jiménez, J. (2020). The association between sleep efficiency and physical performance in taekwondo university students. Retos, 37, 227-232. https://doi.org/10.47197/retos.v37i37.69860

Carrier, J., Sembad, K., Deurveilherd, S., Drogosg, L., Lorda, C. y Sekerovick, Z. (2017). Sex differences in age-related changes in the sleep-wake cycle. Frontiers in Neuroendocrinology, 47, 65-85. https://doi.org/10.1016/j.yfrne.2017.07.004

Chennaoui, M., Arnal, P. J., Sauvet, F. y Léger, D. (2015). Sleep and exercise: A reciprocal issue? Sleep Medicine Reviews, 20, 59-72.

https://doi.org/10.1016/j.smrv.2014.06.008

Revista de Investigación Cuerpo, Cultura y Movimiento

ISSN: 2248-4418 | e-ISSN: 2422-474X | DOI: https://doi.org/10.15332/2422474X

Vol. 11 N. ${ }^{\circ} 1$ | enero-junio de 2021 
Copenhaver, E. A. y Diamond, A. B. (2017). The value of sleep on athletic performance, injury, and recovery in the young athlete. Pediatric annals, 46(3), e106-e111. https://doi.org/10.3928/19382359-20170221-01

Fullagar, H. H., Duffield, R., Skorski, S., Coutts, A. J., Julian, R. y Meyer, T. (2015). Sleep and Recovery in Team Sport: Current Sleep-Related Issues Facing Professional Team-Sport Athletes. Int J Sports Physiol Perform, 1O(8), 950-957. https://doi.org/10.1123/ijspp.2014-0565

Fullagar, H. H., Skorski, S., Duffield, R., Hammes, D., Coutts, A. J. y Meyer, T. (2015). Sleep and athletic performance: the effects of sleep loss on exercise performance, and physiological and cognitive responses to exercise. Sports Med, 45(2), 161-186. https://doi.org/10.1007/s40279-014-0260-0

Gómez-González, B., Domínguesz-Salazar, E., Hurtado-Alvarado, G., Esqueda-León, E., Santana-Miranda, R., Rojas-Zamorano, J. A. y Velázquez-Moctezuma, J. (2012). Role of sleep in the regulation of the immune system and the pituitary hormones. Annals of the New York Academy of Sciences, 1261(1), 97-106. https://doi.org/10.1111/j.1749-6632.2012.06616.x

Halson, S.L. (2014a). Monitoring Training Load to Understand Fatigue in Athletes. Sports Medicine, 44(Suppl 2), S139-S147. https://doi.org/10.1007/s40279-014$\underline{0253-\mathrm{Z}}$

Halson, S.L. (2014b). Sleep in elite athletes and nutritional interventions to enhance sleep. Sports Med, 44 (Suppl 1), S13-23. https://doi.org/10.1007/s40279-014$\underline{0147-0}$

Keramidas, M., Gadefors, M., Nilsson, L. O. y Eiken, O. (2018). Physiological and psychological determinants of whole-body endurance exercise following short-term sustained operations with partial sleep deprivation. European Journal of Applied Physiology, 118, 1373-1384. https://doi.org/10.1007/s00421-018-3869-0

Ketema, P., Dugovic, C., Turek, F. y Laposky, A. (2006). Diurnal Sex Differences in the Sleep-Wake Cycle of Mice are Dependent on Gonadal Function. Sleep, 29(9), 12111223. https://doi.org/10.1093/sleep/29.9.1211

Leeder, J., Glaister, M., Pizzoferro, K., Dawson, J. y Pedlar, C. (2012). Sleep duration and quality in elite athletes measured using wristwatch actigraphy. Journal of Sports Sciences, 3o(6), 541-545. https://doi.org/10.1080/02640414.2012.660188 
Mah, C. D., Mah, K. E., Kezirian, E. J. y Dement, W. C. (2011). The effects of sleep extension on the athletic performance of collegiate basketball players. Sleep, 34(7), 943-950. https://doi.org/10.5665/sleep.1132

Milewski, M., Skaggs, D., Bishop, G., Pace, J., Ibrahim, D., Wren, T. y Barzdukas, A. (2014). Chronic lack of sleep is associated with increased sports injuries in adolescent athletes. Journal of Pediatric Orthopaedics, 34(2), 129-133. https://doi.org/10.1097/bpo.0000000000000151

Mong, J. y Cusmano, D. (2015). Sex differences in sleep: impact of biological sex and sex steroids. Philosophical Transactions B Royal Society, 371(1688), 1-9. https://doi.org/10.1098/rstb.2015.0110

Peake, J. M., Neubauer, O., Walsh, N. P. y Simpson, R. J. (2016). Recovery of the immune system after exercise. Journal of Applied Physiology, 122 (5), 1077-1087. https://doi.org/10.1152/japplphysiol.00622.2016

Reeve, S., Sheaves, B. y Freeman, D. (2018). Sleep Disorders in Early Psychosis: Incidence, Severity, and Association with Clinical Symptoms. Schizophrenia Bulletin, 45(2), 287-295. https://doi.org/10.1093/schbul/sby129

Saner, N. J., Lee, M. J. C., Pitchford, N. W., Kuang, J., Roach, G. D., Garnham, A., Stokes, T., Phillips, S., Bishop, J. y Bartlett, J. D. (2020). The effect of sleep restriction, with or without high- intensity interval exercise, on myofibrillar protein synthesis in healthy young men. The Journal of physiology, 598(8), 1523-1536.

https://doi.org/10.1113/jp278828

Sargent, C., Lastella, M., Halson, S. y Roach, G. (2014). The impact of training schedules on the sleep and fatigue of elite athletes. Chronobiology International, 31(10), 1160-1168. https://doi.org/10.3109/07420528.2014.957306

Sargent, C., Lastella, M., Halson, S., y Roach, G. (2016). The validity of activity monitors for measuring sleep in elite athletes. Journal of Science and Medicine in Sport, 19, 848-853. https://doi.org/10.1016/j.jsams.2015.12.007

Schwartz, J. y Simon, R. (2015). Sleep extension improves serving accuracy: A study with college varsity tennis players. Physiology \& Behavior, 151, 541-544.

https://doi.org/10.1016/j.physbeh.2015.08.035

Revista de Investigación Cuerpo, Cultura y Movimiento

ISSN: 2248-4418 | e-ISSN: 2422-474X | DOI: https://doi.org/10.15332/2422474X

Vol. 11 N.o 1 | enero-junio de 2021 
Simpson, N. S., Gibbs, E. L. y Matheson, G. O. (2017). Optimizing sleep to maximize performance: implications and recommendations for elite athletes. Scand J Med Sci Sports, 27(3), 266-274. https://doi.org/10.1111/sms.12703

Skein, M., Duffield, R., Minett, G., Snape, A. y Murphy, A. (2013). The Effect of Overnight Sleep Deprivation After Competitive Rugby League Matches on Postmatch Physiological and Perceptual Recovery. International Journal of Sports Physiology and Performance, 8, 556-564. https://doi.org/10.1123/ijspp.8.5.556

Taylor, S., Rogers, G. y Driver, H. (1997). Effects of training volume on sleep, psychological, and selected physiological profiles of elite female swimmers. Medicine \& Science in Sports \& Exercise, 29(5), 688-693. https://doi.org/10.1097/00005768-199705000-00016

Tuomilehto, H., Vuorinen, V. P., Penttilä, E., Kivimäki, M., Vuorenmaa, M., Venojärvi, M., Airaksinen, O. y Pihlajamäki, J. (2017). Sleep of professional athletes: Underexploited potential to improve health and performance. Journal of Sports Sciences, 35(7), 704-710. https://doi.org/10.1080/02640414.2016.1184300

Van Der Werf, Y. D., Altena, E., Schoonheim, M. M., Sanz-Arigita, E. J., Vis, J. C., De Rijke, W. y Van Someren, E. J. W. (2009). Sleep benefits subsequent hippocampal functioning. Nature Neuroscience, 12(2), 122-123.

\section{https://doi.org/10.1038/nn.2253}

Vyazovskiy, V.V. (2015). Sleep, recovery, and metaregulation: explaining the benefits of sleep. Nature and science of sleep, 7, 171-184. https://doi.org/10.2147/nss.s54036

Walsh, J., Sanders, D., Hamilton, D. y Walshe, I. (2019). Sleep profiles of elite swimmers during different training phases. Journal of Strength and Conditioning Research, 33(3), 811-818. https://doi.org/10.1519/jsc.0000000000002866

Walsh, N. P., Halson, S. L., Sargent, C., Roach, G. D., Nédélec, M., Gupta, L., Leeder, J., Fullagar, H. H., Coutts, A. J., Edwards, B. J., Pullinger, S. A., Robertson, C. M., Burniston, J. G., Lastella, M., Le Meur, Y., Hausswirth, C., Bender, A. M., Grandner, M. A. y Samuels, C. H. (2020). Sleep and the athlete: narrative review and 2021 expert consensus recommendations. British Journal of Sports Medicine, bjsports-2020-102025. https://doi.org/10.1136/bjsports-2020-102025

Revista de Investigación Cuerpo, Cultura y Movimiento ISSN: 2248-4418 | e-ISSN: 2422-474X | DOI: https://doi.org/10.15332/2422474X Vol. 11 N.o 1 | enero-junio de 2021 
Watson, N.F., Badr, M.S., Belenky, G., Bliwise, D.L., Buxton, O.M., Kushida, C., Malhotra, M., Martin, J., Patel, S., Quan, S. y Tasali, E. (2015). Joint Consensus Statement of the American Academy of Sleep Medicine and Sleep Research Society on the Recommended Amount of Sleep for a Healthy Adult: Methodology and Discussion. Sleep, 38(8), 1161-1183. https://doi.org/10.5665/sleep.4716

Xu, W., Tan, C. C., Zou, J. J., Cao, X. P. y Tan, L. (2020). Sleep problems and risk of allcause cognitive decline or dementia: an updated systematic review and metaanalysis. J Neurol Neurosurg Psychiatry, 91(3), 236-244. https://doi.org/10.1136/jnnp-2019-321896

Zhang, Y., Ren, R., Lei, F., Zhou, J., Zhang, J., Wing, Y.-K., Sanford, L. D. y Tang, X. (2019). Worldwide and regional prevalence rates of co-occurrence of insomnia and insomnia symptoms with obstructive sleep apnea: A systematic review and metaanalysis. Sleep Medicine Reviews, 45, 1-17.

https://doi.org/10.1016/j.smrv.2019.01.004 